\title{
In memoria di Arrigo Castellani a 100 anni dalla nascita
}

\author{
Leonardo Castellani
}

PUBBLICATO: 31 DECEMBER 2020

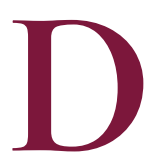

esidero esprimere innanzitutto, a nome della mamma Ornella, delle sorelle Ilaria e Elena, e del nipote Beniamino, i ringraziamenti alla Crusca per questa toccante iniziativa in ricordo di Arrigo Castellani. Grazie al presidente Claudio Marazzini, a Valeria Della valle e Pär Larson per i loro emozionanti e interessanti interventi. Questa iniziativa ci fa sentire oggi, come I6 anni fa, quando nella villa medicea sede della Crusca fu dato l'addio a nostro padre, l'affetto di una comunità di colleghi, allievi e amici del babbo.

La Crusca, i cruscanti, le tornate, sono parole che abbiamo sentito in casa fin da bambini, diventate parte del lessico di famiglia.

Chissà cosa direbbe il babbo oggi, a vederci girare con le mascherine e parlare dentro a un computiere per fare seminari e lezioni a distanza. Di certo avrebbe sostituito confinamento a lockdown (come del resto fanno i francesi, che fin dall'inizio hanno usato "confinement") ma probabilmente avrebbe finito con l'usare anche lui un calcolatore (termine tollerato, anche se era preferito computiere) per comunicare con studenti e colleghi.

Non era ostile alla tecnologia, tutt'altro. Ne ha fatto uso spesso per i suoi studi. Mi ricordo ad esempio che per le sue ricerche di fonetica si appoggiava a risultati di apparati che fornivano sonogrammi e spettrogrammi dei fonemi, e riproduzioni di disegni e radiografie degli organi fonatori. Per noi erano divertenti le sue registrazioni dei fonemi, ascoltate al contrario su un magnetofono (bastava avvolgere il nastro al contrario) per evidenziare caratteristiche che rimanevano nascoste nell' ascolto normale.

Era figlio di un ingegnere, nostro nonno Claudio Castellani, costruttore di diverse centrali elettriche del Nord Italia, e il metodo e la mentalità scientifica facevano parte del suo carattere di studioso. Con l'aiuto del padre Claudio aveva ricostruito negli anni 60 una formula di analisi combinatoria che gli serviva per individuare la più probabile gerarchia temporale di manoscritti. Molti anni dopo mi telefonò - abitavo già a Torino - per chiedermi come modificarla per adattarla a un altro caso che stava studiando.

I suoi interessi scientifici potevano prendere anche una direzione più frivola, e mi riferisco ai suoi momenti di svago con i libercoli (come li chiamava) di fantascienza, di cui era un cultore appassionato. Il risultato di questa passione era una vasta collezione, che comprendeva gran parte della produzione di fantascienza in lingua inglese dagli anni 50 in poi. I libercoli poi erano anche libri gialli e di spionaggio, sempre in inglese. Qui credo che fossero le sue memorie di guerra ad esserne sollecitate. Comunque sia, su questa parte della biblioteca paterna ho imparato l' inglese. Spesso scambiavamo pareri sull' ultimo libercolo letto, e ogni tanto contribuivo anch'io alla collezione con qualche libercolo nuovo.

La sua macchina da scrivere, una Lettera 22, con il suo ticchettio che ci accompagnava da piccoli nel primo sonno, era diventata ormai obsoleta, e si faceva fatica trovare i nastri inchiostrati. Gli regalammo allora, erano i primi anni novanta (anzi Ilaria mi dice esattamente nel i99o, per i suoi 70 
anni), un portatile, uno dei primi modelli, assai ingombrante rispetto a quelli attuali. Con pazienza ne imparò il funzionamento, e lo usò per scriverci un articolo. Ma l'esperienza non dovette convincerlo del tutto, perché poi tornò alla gloriosa Lettera 22. La mamma allora si impossessò del portatile, e divenne presto abbastanza esperta, usandolo per molti suoi lavori.

Non era raro che il babbo approfittasse della dimestichezza della mamma col computiere per chiederle di assisterlo in certe sue ricerche (ricordo ad esempio statistiche delle concordanze). Noi figli le facevamo da consulenti per i nuovi portatili che via via sostituivano quelli vecchi.

La forma mentis scientifica deve aver giocato un ruolo anche nel suo interesse per l' esperanto. Nostro padre ne ammirava la struttura logica e semplice, e le sue notevoli potenzialità espressive. Forse anche il polacco, che conosceva bene per ragioni di studio (era stato in Polonia con una borsa di studio) e di guerra (aveva in seguito passato 2 anni in Polonia come ufficiale-interprete, ricordato da da Pär Larson) può aver contribuito a questo suo interesse: l' ideatore Zamenhof essendo polacco, l' esperanto ne contiene diverse tracce. Se ne occupò in un articolo, e credo in alcuni discorsi tenuti in occasione dei roo anni dell' esperanto. Ho conservato grammatiche e alcuni testi in esperanto. Se vi capita, potete guardare in rete dei filmati di ragazzi, esperantisti nativi (figli di genitori esperantisti), che parlano tra di loro in esperanto con la massima naturalezza. E' quasi tutto comprensibile senza studio, ed è divertente cogliere gli accenti delle loro lingue nazionali.

Nel suo articolo l'esperanto viene contrapposto all' inglese, lingua ormai universale per la letteratura scientifica e sempre più presente nell' uso quotidiano, nei giornali, in televisione, in rete. Nella maggior parte dei casi gli anglicismi appaiono inutili e facilmente sostituibili con parole italiane, o con adattamenti e neologismi che rispettino la struttura linguistica dell' italiano, come il babbo Arrigo predicava ai tempi del suo articolo Morbus Anglicus delig87. I suoi neologismi, tutti dottamente motivati, erano a volte abbastanza buffi e oggetto di gran divertimento in famiglia, e anche di emulazione. Non esitava a usarli nella pratica: dal panettiere sotto casa chiedeva sempre i "cracchieri" e agli amici poteva offrire un bicchierino di "guisco".

Riguardo al polacco aggiungo questo: non ho memoria di aver mai sentito il babbo imprecare con male parole, tranne in polacco con parole dal suono molto minaccioso come "psciacreff!" (sangue d'un cane). La sua signorilità innata si manifestava anche nelle arrabbiature più forti: a un collega con cui litigò per le solite ragioni di concorsi universitari, lo sentii (quasi) urlare al telefono, prima di buttar giù la cornetta: "tu non sei un gentiluomo! ".

Vorrei concludere ricordando il sodalizio tra i nostri genitori, Ornella e Enrico (Arrigo), non solo compagni di una vita, ma anche "compagni di riva" (come scrisse nostra madre nella dedica del suo libro "In riva al fume della lingua"), innamorati entrambi della lingua e della sua storia. Non capita a tutti di avere due genitori accademici della Crusca e di assistere ad accese discussioni linguistiche durante i pasti, con movimentate consultazioni del DOP e del DEI. Il babbo era capace di fare le scale su e giù più volte durante un pasto, per appurare come stessero le cose. In qualche modo, per osmosi, credo che sensibilità e gusto per la lingua si siano tramandati ai figli, che svolgono professioni in ambito scientifico, e al nipote Beniamino (figlio di Elena), che avrebbe senza dubbio dato grande soddisfazione al nonno per i suoi molteplici interessi letterari e anche bibliofilici.

Uno dei rimpianti del babbo era che nessuno di noi tre figli, che pure manifestavamo un certo gusto per la lingua, avesse imboccato la via tracciata da lui e dalla mamma. Questo rimpianto fu però addolcito dalla vocazione della nipote Valentina, figlia di un fratello di Ornella, il cui legame con lo zio si fece sempre più stretto da quando lei diventò ricercatrice presso l' Opera del Vocabolario (all'Accademia delle Crusca). Spesso zio e nipote si consultavano l'un l'altra, parlando per lunghe ore 
al telefono o nel salotto di casa, e il babbo manifestò sempre un'affetto e una tenerezza particolare per Valentina. La triste sorte volle che lei venisse a mancare solo qualche settimana dopo di lui, dopo lunghi anni di malattia, a soli 47 anni. In questa occasione in cui ricordiamo il babbo, ci sembra naturale ricordare anche la nostra cara cugina Valentina, che molti di voi hanno avuto occasione di conoscere ed apprezzare. Grazie per la vostra attenzione.

\section{Cita come:}

Leonardo Castellani, In memoria di Arrigo Castellani a 100 anni dalla nascita , "Italiano digitale", 2020, XV, 2020/4 (ottobre-dicembre)

DOI: $10.35948 / 2532-9006 / 2021.5444$

Copyright 2020 Accademia della Crusca

Pubblicato con licenza creative commons CC BY-NC-ND 